\title{
Migração pendular e saúde: perfil de paraguaios em tratamento dialítico em município de fronteira internacional
}

\author{
Pendular migration and health: profile of \\ Paraguayans undergoing dialysis treatment \\ in an international border municipality
}

\section{Ederson Pereira' ${ }^{1}$ (D) Manoela de Carvalho² (D)}

'Autor para correspondência. Universidade Estadual do Oeste do Paraná (Cascavel). Paraná, Brasil. edernefro@gmail.com 2Universidade Estadual do Oeste do Paraná (Cascavel). Paraná, Brasil. manoelacarv@gmail.com

\begin{abstract}
RESUMO | OBJETIVO: Caracterizar o perfil sociodemográfico e clínico de pacientes de origem paraguaia em tratamento de hemodiálise em Foz do Iguaçu, Paraná. MÉTODO: Pesquisa exploratória e descritiva realizada com 23 pacientes paraguaios cujos dados foram extraídos do banco de dados do serviço especializado de nefrologia credenciado ao SUS. Os dados foram analisados por estatística descritiva. RESULTADOS: Em relação ao perfil sociodemográfico, observou-se que $73,9 \%$ eram do sexo masculino, $65,2 \%$ casados, $26,1 \%$ com ensino fundamental incompleto, $43,45 \%$ estavam na faixa etária de 40 a 59 anos e $47,85 \%$ com idade $\geq 60$ anos. Quanto às características clínicas, 73,9\% usaram cateter como primeiro acesso e $78,3 \%$ realizaram a primeira sessão de hemodiálise em caráter de emergência; a diabetes e a hipertensão foram as principais doenças de bases e $39,2 \%$ não estavam sob cuidados de nefrologista na fase não dialítica. CONCLUSÃo: 0 perfil observado foi, em sua maioria, homens na faixa etária acima de 50 anos, que vive com companheira(o) e filhos, que tiveram o SUS como financiador do tratamento e iniciaram tardiamente o tratamento, em caráter emergencial. Em razão desses achados, sugere-se a disponibilidade de serviços de apoio aos familiares e acompanhantes, além do fortalecimento da Atenção Básica nesses territórios.
\end{abstract}

DESCRITORES: Áreas de Fronteira. Diálise Renal. Doença Crônica. Atenção à Saúde. Sistema Único de Saúde.

\begin{abstract}
OBJECTIVE: To characterize the sociodemographic and clinical profile of patients of Paraguayan origin on the hemodialysis treatment in Foz do Iguaçu, Paraná. METHOD: exploratory and descriptive research was carried out with 23 Paraguayan patients whose data were extracted from the specialized nephrology service accredited to the UHS database. Descriptive statistics analyzed the data. RESULTS: regarding the socio-demographic profile, it was observed that $73.9 \%$ were male, $65.2 \%$ married, $26.1 \%$ with incomplete elementary education, $43.45 \%$ were in the age range of 40 to 59 years, and $47.85 \%$ aged $\geq 60$ years. As for the clinical characteristics, $73.9 \%$ used catheters as their first access, and $78.3 \%$ performed their first hemodialysis session on an emergency basis; diabetes and hypertension were the main underlying diseases, and $39.2 \%$ were not under nephrologist care in the non-dialytic phase. CONCLUSION: the profile observed was mostly men over 50 years old, living with a partner and children, who had the UHS as a funder of treatment and started treatment late, on an emergency basis. Because of these findings, the availability of support services for family members and companions is suggested, and the strengthening of Primary Care in these territories.
\end{abstract}

DESCRIPTORS: Border Areas. Renal Dialysis. Chronic disease. Health Care. Unified Health System. 


\section{Introdução}

O aumento da prevalência da doença renal crônica (DRC) e, consequentemente, sua repercussão na morbimortalidade dos acometidos tornaram-se um problema mundial de saúde pública. ${ }^{1} \mathrm{Em}$ diversos países, estima-se uma prevalência de $8 \%$ a $16 \%$ de pessoas com algum grau de comprometimento da função renal, constituindo futuramente um grande grupo de indivíduos que necessitarão de terapia renal substitutiva (TRS), caso ocorra a progressão desta disfunção, evoluindo para as fases finais da DRC. $\underline{\text { ? }}$

Regiões de fronteira constituem um recorte analítico e espacial de múltiplas realidades sociais, políticas, econômicas e culturais, e podem se configurar espaço de conflitos transculturais e identitários. Nesses espaços, o local e o internacional se articulam e estabelecem dinâmicas próprias, construídas pelos povos fronteiriços, que tornam a fronteira uma forma diferenciada de organização territorial no bojo da ordem capitalista, recriando também outros aspectos regionais. As políticas para ordenamento territorial bilaterais ou multilaterais repercutem nos fluxos populacionais nessas regiões, pois não há territórios sem sujeitos e, portanto, todo território se faz por meio dos sujeitos sociais. 3

Com a aprovação da Lei Federal no 13.445, em 2017, é garantida aos residentes fronteiriços a mesma atenção à saúde no Sistema Único de Saúde (SUS) que os brasileiros possuem. Os residentes fronteiriços são pessoas que vivem em países vizinhos, numa área de forte interação social, econômica, política e cultural com os brasileiros, mas não são considerados migrantes, já que não cruzam a fronteira de um país para outro com o objetivo de fixar-se no outro país, pois conservam suas residências habituais no país de origem. ${ }^{4}$

A migração pendular pode ser definida como movimentos cotidianos dos residentes fronteiriços entre o local de residência e locais de trabalho ou estudo, especialmente quando essa mobilidade implica em residir em certo município e trabalhar ou estudar em outro, não existindo uma troca definitiva de local de residência. Esse tipo de mobilidade também ocorre em regiões fronteiriças para buscar atendimento em saúde, influenciando no planejamento de serviços e ações, visto que a adesão ao tratamento e o prognóstico dos pacientes em mobilidade pendular são impactados por diagnósticos tardios e redução do acesso. ${ }^{5-6}$
No SUS, essa mobilidade pendular para atendimento em saúde está prevista legalmente na Lei Federal $n^{\circ} 8.080$ de 1990 quando enfatiza a organização regionalizada do sistema, prevendo que os municípios estabeleçam mecanismos, como os consórcios intermunicipais para ofertar ações e serviços conjuntamente. Mais recentemente, o Decreto $n^{\circ} 7.508$ de 2011 regulamenta os papéis dos entes federativos nas regiões e redes de saúde, e define região de saúde como espaço geográfico contínuo constituído por agrupamentos de municípios limítrofes, delimitado a partir de identidades culturais, econômicas e sociais, e de redes de comunicação e infraestrutura de transportes compartilhados, com a finalidade de integrar a organização, bem como planejamento e a execução de ações em saúde.?

No entanto, a migração pendular para fins de uso público dos serviços de saúde torna-se mais complexa quando se considera a mobilidade internacional, ou seja, quando dois países têm fronteiras entre si e as disparidades sociais e econômicas determinam diferenças na oferta e qualidade de serviços de

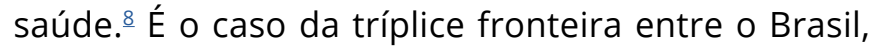
Paraguai e Argentina, onde o município de Foz do Iguaçu atende cotidianamente um fluxo populacional que excede a base populacional sobre a qual é calculado o repasse de verbas para o sistema municipal, o que implica em dificuldades financeiras para a manutenção dos serviços de saúde e no estabelecimento de barreiras para o acesso dos fronteiriços não residentes a estes serviços. $\frac{5}{}$

Entende-se que o problema do subfinanciamento do SUS não resulta da assistência aos residentes fronteiriços não nacionais nesses municípios, haja vista que a falta de recursos financeiros para o SUS não é observada apenas em municípios fronteiriços. No entanto, essa condição motiva as gestões municipais a viabilizarem ao estrangeiro atendimento em saúde nos casos de urgência, emergência e na atenção primária, mas restringirem o acesso dos fronteiriços não nacionais a serviços especializados de maior complexidade. . $^{5,-10}$ Ainda assim, muito residentes fronteiriços não nacionais conseguem acessar assistência da alta complexidade pelo SUS nos municípios de fronteira, para doenças crônicas, como é o caso da hemodiálise.

Foz do Iguaçu tem registrado importante elevação da população flutuante composta por migrantes, turistas e estudantes, tanto do país quanto do exterior, 
que busca atendimento no sistema público de saúde brasileiro. ${ }^{11}$ Estima-se que no Paraguai existam 176 pacientes em diálise por milhão de habitantes, sendo um dos países com menor cobertura deste serviço, com somente 1.184 pacientes com Insuficiência Renal Crônica (IRC) que recebem tratamento de hemodiálise em todo o país. $\frac{12}{}$

Ao contrário do sistema de saúde brasileiro, que pressupõe a universalidade do acesso e a integralidade da assistência, assegurando o tratamento dialítico, mesmo que ofertado em serviços privados, o sistema de saúde do Paraguai é subdividido em público, com baixa cobertura, e privado, cujos serviços médico-previdenciários representam 21,6\% de abrangência da assistência. O sistema de saúde paraguaio apresenta elevada segmentação de prestadores de serviços e não garante a cobertura total no tratamento para pessoas com IRC. $\frac{13}{}$

Nesse cenário, onde as dificuldades encontradas por pacientes paraguaios para o tratamento em seu país de origem favorecem a busca de serviços no Brasil, objetivou-se caracterizar o perfil sociodemográfico e clínico de pacientes de nacionalidade paraguaia em tratamento de hemodiálise de um serviço de referência para nefrologia em Foz do Iguaçu, Paraná.

\section{Método}

Pesquisa descritiva de abordagem quantitativa realizada em um serviço privado de diálise conveniado ao Sistema Único de Saúde (SUS), referência em alta complexidade para nefrologia, situado em Foz do Iguaçu, município brasileiro pertencente ao estado do Paraná, fronteira com Argentina e Paraguai.

O serviço referenciado como lócus do estudo iniciou as atividades no ano de 1988, ofertando serviço de terapia renal substitutiva (TRS), a hemodiálise e a diálise peritoneal, além de atendimento clínico ambulatorial de pacientes em tratamento conservador e, também, transplantados renais. Em janeiro de 2018, este serviço atendia 269 pacientes em hemodiálise e 23 pacientes em diálise peritoneal, totalizando 292 pacientes em tratamento dialítico; destes, $23(8 \%)$ indivíduos eram de nacionalidade paraguaia, e todos realizavam hemodiálise.
A população do estudo foi a totalidade dos pacientes de nacionalidade paraguaia $(n=23)$ cadastrados como ativos no serviço em tratamento dialítico. A coleta dos dados ocorreu nos meses de fevereiro e abril de 2018.

Os dados relacionados ao perfil sociodemográfico (sexo, idade, estado civil, com quem vive, presença de filhos, escolaridade, vínculo empregatício, convênio de saúde) e clínico (início do tratamento, local da primeira sessão, tipo de acesso na primeira sessão, doenças de base, acesso ao nefrologista) foram coletados dos prontuários eletrônicos por meio do Programa NephroSys. Tais informações estão disponíveis no lócus da pesquisa, incluindo evolução clínica, dados demográficos e socioeconômicos.

Os dados foram organizados em planilhas no programa Excel e posteriormente analisados por meio da estatística descritiva e apresentados na forma de tabelas. Este estudo foi aprovado pelo Comitê de Ética em Pesquisa em Seres Humanos.

\section{Resultados}

A Tabela 1 caracteriza os pacientes segundo sexo, estado civil, presença de filhos, com quem vive e escolaridade. Entre os paraguaios em tratamento, destaca-se que $73,90 \%(n=17)$ são homens, $87,3 \%(n=18)$ têm mais de 50 anos de idade, 69,56\% ( $n=16)$ possuem e vivem com companheiro(a), a maioria possui filhos $(78,30 \%)$ mas, $56,5 \%(n=13)$ vivem com filhos, ressaltando que esta variável admitia mais de uma resposta.

O vínculo empregatício, importante informação para construção de indicadores acerca da determinação social do processo saúde-doença e da relação entre o trabalho e o adoecimento, não estava registrada nos prontuários de $87,10 \%(n=20)$ dos pacientes. Quanto ao deslocamento do domicílio à clínica de nefrologia, 34,8\% ( $n=08)$ usam carro próprio e $26,0 \%(n=06)$ transporte público para se locomover até a unidade de diálise. O SUS foi o principal financiador do tratamento para $82,6 \%(n=19)$ dos pacientes paraguaios, conforme apresenta a tabela 1. 
Tabela 1. Caracterização sociodemográfica dos pacientes paraguaios em tratamento dialítico. Foz do lguaçu, PR, Brasil, 2018. (N=23)

\begin{tabular}{|c|c|c|}
\hline Variáveis & $\mathbf{n}$ & $\%$ \\
\hline \multicolumn{3}{|l|}{ Sexo } \\
\hline Masculino & 17 & 73,90 \\
\hline Feminino & 06 & 26,10 \\
\hline \multicolumn{3}{|l|}{ Faixa etária (anos) } \\
\hline 30-39 anos & 02 & 8,70 \\
\hline 40-49 anos & 03 & 13,00 \\
\hline $50-59$ anos & 07 & 30,45 \\
\hline$\geq 60$ anos & 11 & 47,85 \\
\hline \multicolumn{3}{|l|}{ Estado Civil } \\
\hline Sem companheiro(a) - solteiro(a), divorciado(a), viúvo(a) & 06 & 26,10 \\
\hline Com companheiro(a) - casado (a), união consensual & 16 & 69,56 \\
\hline Não consta informação & 01 & 4,35 \\
\hline \multicolumn{3}{|l|}{ Filhos } \\
\hline Sim & 18 & 78,30 \\
\hline Não & 04 & 17,40 \\
\hline Não consta informação & 01 & 4,30 \\
\hline \multicolumn{3}{|l|}{ Vive no mesmo domicílio } \\
\hline Pais & 01 & 4,30 \\
\hline Filhos & 13 & 56,50 \\
\hline Marido/Esposa & 16 & 69,56 \\
\hline Sozinho & 01 & 4,30 \\
\hline \multicolumn{3}{|l|}{ Escolaridade } \\
\hline Ensino Fundamental Incompleto & 06 & 26,10 \\
\hline Ensino Superior Completo & 01 & 4,30 \\
\hline Não consta informação & 16 & 69,60 \\
\hline \multicolumn{3}{|l|}{ Vínculo Empregatício } \\
\hline Empregado & 01 & 4,30 \\
\hline Autônomo & 01 & 4,30 \\
\hline Aposentado & 01 & 4,30 \\
\hline Sem registro & 20 & 87,10 \\
\hline \multicolumn{3}{|l|}{ Deslocamento até a clínica } \\
\hline Transporte coletivo urbano & 04 & 17,40 \\
\hline Transporte coletivo intermunicipal & 02 & 8,70 \\
\hline Carro próprio & 08 & 34,80 \\
\hline Carona & 03 & 13,00 \\
\hline Sem registro & 06 & 26,10 \\
\hline \multicolumn{3}{|l|}{ Tipo de Convênio de Saúde } \\
\hline SUS & 19 & 82,60 \\
\hline Privado & 04 & 17,40 \\
\hline
\end{tabular}

Fonte: Sistema de Informação NephroSys, 2018. 
Quanto à caracterização clínica, sobre o local da primeira sessão de hemodiálise, verificou-se que 56,5\% ( $n=13$ ) dos pacientes paraguaios iniciaram tratamento de hemodiálise na clínica de hemodiálise. No que se refere ao primeiro tipo de acesso vascular, observou-se a fístula arteriovenosa para $17,4 \%(n=04)$ e que $91,3 \%(n=21)$ iniciaram o tratamento dialítico com mais de 40 anos e, ainda, que 60,90\%\% ( $n=14)$ tinham de 1 a 4 anos de tratamento. Já o uso de cateter como primeiro acesso ocorreu em 73,9\% ( $n=17)$ dos pacientes. O estudo identificou que $78,3 \%$ $(n=18)$ realizaram a primeira sessão de hemodiálise em caráter de emergência. As duas principais doenças de bases foram diabetes mellitus em 73,9\% ( $n=17)$ e hipertensão arterial sistêmica em $65,2 \%(n=15)$. Antes de iniciar o tratamento de hemodiálise, 39,2\% ( $n=09$ ) deles não estavam sob cuidados de nefrologista (Tabela 2).

Tabela 2. Caracterização clínica e de tratamento dos pacientes paraguaios em tratamento de hemodiálise. Foz do Iguaçu, PR, Brasil, 2018. (N=23)

\begin{tabular}{|c|c|c|}
\hline Variáveis & $\mathbf{N}$ & $\%$ \\
\hline \multicolumn{3}{|c|}{ Local da primeira sessão de hemodiálise } \\
\hline Clínica de hemodiálise & 13 & 56,50 \\
\hline Hospital & 08 & 34,80 \\
\hline Não consta informação & 02 & 8,70 \\
\hline \multicolumn{3}{|c|}{ Faixa etária em que iniciou tratamento } \\
\hline 30-39 anos & 05 & 21,70 \\
\hline $40-49$ anos & 02 & 8,70 \\
\hline $50-59$ anos & 10 & 43,50 \\
\hline$\geq 60$ anos & 06 & 26,10 \\
\hline \multicolumn{3}{|l|}{ Tempo de tratamento } \\
\hline$<1$ ano & 02 & 8,70 \\
\hline 1 a 4 anos & 14 & 60,90 \\
\hline$\geq 5$ anos & 07 & 30,43 \\
\hline \multicolumn{3}{|l|}{ Primeiro tipo de acesso } \\
\hline Fístula Arteriovenosa & 04 & 17,40 \\
\hline Cateter & 17 & 73,90 \\
\hline Não consta informação & 02 & 8,70 \\
\hline \multicolumn{3}{|c|}{ Caráter da primeira sessão de hemodiálise } \\
\hline Eletiva & 03 & 13,00 \\
\hline Emergência & 18 & 78,30 \\
\hline Não consta informação & 02 & 8,70 \\
\hline \multicolumn{3}{|l|}{ Doença de Base } \\
\hline Hipertensão Arterial & 15 & 65,20 \\
\hline Diabetes Mellitus & 17 & 73,90 \\
\hline Doença Cardiovascular & 04 & 17,40 \\
\hline \multicolumn{3}{|l|}{ Sob cuidados de nefrologista } \\
\hline Não & 09 & 39,20 \\
\hline Sim - há menos de 06 meses & 02 & 8,70 \\
\hline Sim - entre 06 e 12 meses & 08 & 34,80 \\
\hline Sim - há mais de 12 meses & 03 & 13,00 \\
\hline Não consta informação & 01 & 4,30 \\
\hline
\end{tabular}

Fonte: Sistema de Informação NephroSys, 2018. 


\section{Discussão}

A predominância de homens acima dos 40 anos, casados e com baixa escolaridade entre os sujeitos pesquisados também foi identificada em outros estudos. ${ }^{14-16}$ Há distintos níveis de risco para doenças crônicas entre homens e mulheres, considerando que entre os gêneros há iniquidades nas condições de trabalho e de vida que os expõem a outros fatores, como estresse, dependência química (álcool e tabaco), excesso de peso e ingesta alimentar rica em gordura. ${ }^{17}$ É importante destacar que o fato de a maioria dos paraguaios realizar tratamento dialítico em outro país, ser casado e ter filhos pode interferir na dinâmica e no apoio familiar desses sujeitos, uma vez que o tratamento é longo e distante da residência.

O perfil socioeconômico dos pacientes em tratamento dialítico é pouco abordado nos estudos epidemiológicos de estrangeiros ou migrantes, sendo insuficientemente estudada a relação entre a ocupação, tipo de vínculo empregatício e o adoecimento renal. Percebe-se, também, o problema da subnotificação desta informação nos prontuários, dificultando análises acerca da determinação social do adoecimento. Outros estudos 5,9 também já apontaram para a falta de registros dos atendimentos prestados aos estrangeiros que dificulta a definição dos perfis dessa população e de estimativas de demandas mais próximas das realidades sociais.

Os documentos requeridos para o atendimento pelo SUS como cartão SUS, cartão da família, comprovante de residência e documento de identidade nacional são uma das principais barreiras enfrentadas pelos residentes fronteiriços não nacionais, uma vez que podem ter seu acesso negado ou obtê-lo com qualidade inferior aos brasileiros. Tal restrição criou uma cultura de fraudar os dados sobre residência, utilizando endereços falsos ou outros recursos para usufruir do atendimento do SUS, consequentemente dificultando a real dimensão da demanda de estrangeiros aos serviços do SUS nesses municípios. $5,-10 \mathrm{~A}$ ausência de informações importantes sobre variáveis relacionadas ao trabalho, ou até mesmo sobre o transporte utilizado para acessar os serviços, dificulta a compreensão da determinação social do adoecimento. Longas distâncias percorridas até os serviços de saúde reduzem o acesso e produzem efeitos sobre a saúde como atraso no diagnóstico e pouca adesão ao tratamento. $\frac{5}{}$
Identificou-se um expressivo percentual de pacientes paraguaios com tempo de tratamento superior a 5 anos, ainda que a maioria apresente tempo de tratamento semelhante a outros estudos realizados no Brasil. ${ }^{17-18}$ No entanto, observa-se que o início do tratamento é tardio entre eles, além de verificar que a clínica de hemodiálise foi o local mais utilizado na primeira sessão de diálise, e que um percentual importante de pacientes realizou a primeira sessão no hospital, corroborando com o caráter de emergência no primeiro tipo de sessão e a necessidade de utilizar cateter central como via de acesso no início do tratamento. Esses resultados sugerem maior dificuldade de acesso à assistência à saúde entre os residentes fronteiriços no Paraguai.

O SUS foi o principal financiador do tratamento dialítico, de acordo com os achados neste estudo e outros, variando de $75,4 \%$ a $86,7 \% \cdot 15-16,18$ Destaca-se a necessidade de sistemas de saúde com integralidade do cuidado coordenado a partir da Atenção Primária para diagnósticos precoces e acompanhamentos adequados das doenças crônicas, como sugerem as Diretrizes Clínicas para o Cuidado ao Paciente com DRC (2014) $)^{19}$ e dos Protocolos de Encaminhamento da Atenção Básica para a Atenção Especializada $\underline{20}$ nos quais uma das especialidades priorizadas foi a nefrologia.

Porém, para a população em estudo, o fato de enfrentarem dificuldades no acesso em razão da distância geográfica e das barreiras organizacionais, a busca ativa e o acompanhamento dos pacientes no território internacional são praticamente inviáveis, tendo em vista a baixa integração entre os serviços de saúde dos dois lados da fronteira apontada em outros estudos.., $8-9$

A hipertensão arterial sistêmica (HAS) e a diabetes mellitus (DM) têm importante predomínio entre as doenças de base dos portadores de DRC, conforme outros estudos. $\frac{15-16}{16}$ Apesar disso, a maioria dos pacientes do presente estudo com HAS e DM não teve acompanhamento conservador com nefrologista. Sujeitos que integram os grupos de risco que predispõem a DRC devem ter acesso a uma abordagem integral a saúde desde os níveis primários de atenção à saúde, realizando o diagnóstico e o acompanhamento nos estágios iniciais da doença, bem como poder acessar especialistas em serviços de média e alta complexidade quando necessário.21-22 
Um dos alicerces do tratamento da DRC é o encaminhamento precoce para consulta com nefrologista, para reduzir a necessidade de TRS. Pacientes encaminhados precocemente para acompanhamento especializado apresentam menor tempo de internação hospitalar após o começo da terapia dialítica e menor taxa de letalidade no primeiro ano de TRS. Além disso, têm menores chances de necessitar de diálise de urgência, melhor controle da pressão arterial e menos edema agudo de pulmão, além de custos menores do tratamento. 22

A partir do perfil dos pacientes paraguaios em tratamento dialítico em município de fronteira internacional propõe-se a reflexão sobre a importância de sistemas nacionais de saúde de acesso universal e integral. As dificuldades de acesso ao tratamento em um lado da fronteira além de se manifestarem no estágio avançado em que os pacientes chegam aos serviços, impactam no prognóstico e nas condições de enfrentamento da doença.

Estudos que descrevem o perfil de pacientes em estágios avançados de doenças crônicas, como no caso do tratamento dialítico, podem ser úteis na definição de programas de prevenção em níveis primários da assistência e, indicam a necessidade de que países fronteiriços harmonizem a legislação de seus sistemas de saúde a fim de garantir oferta universal e integral de serviços para assegurar o direito a ações de prevenção e tratamento restaurador da saúde de seus cidadãos.

Submeter-se a deixar a residência em um país, mesmo que temporariamente, para obter atendimento à saúde em outro, em momento de fragilidade, e enfrentar as incertezas sobre o acesso e a continuidade do tratamento produz impacto na saúde de residentes fronteiriços. Deve, portanto, constituir preocupação não apenas para o paciente e seus familiares, mas aos gestores e profissionais de saúde que formulam políticas públicas, bem como, aos pesquisadores para compreenderem mais profundamente as diferenças nos perfis de adoecimento crônico nesta população. Ressalta-se, ainda, que em regiões de fronteira entre o Brasil e outros países, há necessidade de os estudos identificarem iniciativas e oportunidades loco-regionais de ações integradoras dos serviços que possibilitem minimizar as assimetrias e iniquidades existentes entre os sistemas de saúde.
- Tais considerações permitem apontar que em municípios situados em região de fronteira internacional, o SUS tem se constituído em um importante recurso de saúde para estrangeiros que não dispõem de um sistema de saúde universalidade de acesso e assistência integral, podendo se configurar como experiência singular motivadora de transformações nos sistemas de saúde dos países vizinhos. Em razão desse perfil, sugere-se a disponibilidade de serviços de apoio aos familiares e acompanhantes, como casas de apoio, pois trata-se de serviços disponíveis apenas em algumas localidades específicas do território brasileiro, servindo de referência para municípios menores e outros países, no caso de região de fronteira internacional.

Por fim, cumpre salientar que, por se tratar de estudo com dados secundários, os limites relacionam-se à ausência de registros importantes, como por exemplo, a ocupação dos pacientes atendidos, e outras variáveis que podem esclarecer aspectos relacionados às dificuldades e mudanças no modo de vida, individual e familiar, quando se busca por serviços de saúde em outro país, apontando para a necessidade de outros estudos para maior aprofundamento.

\section{Conclusão}

Por meio desta pesquisa, foi possível conhecer o perfil sociodemográfico e clínico de pacientes de origem paraguaia em tratamento de hemodiálise, serviço de alta complexidade em nefrologia, em um município situado em região de fronteira internacional. Tratase de pacientes, em sua maioria, homens, na faixa etária acima de 50 anos, que vive com companhei$\mathrm{ra}(\mathrm{o})$ e filhos, e que tiveram o SUS como financiador do tratamento. Em relação aos aspectos clínicos, observou-se que a maioria realizava hemodiálise de 2 a 5 anos, o primeiro acesso ocorreu com uso de cateter e a primeira sessão de hemodiálise em caráter de emergência, e ainda, apresentaram diabetes e hipertensão arterial como principais doenças de base e muitos não tinham acompanhamento de nefrologista. Tal situação pode ser explicada por dificuldades no acesso à Atenção Básica, no país de origem, ou ainda, por baixa resolutividade no manejo das doenças de base, que acarreta o início tardio e de caráter emergencial, em estágio bastante avançado da DRC. 
Nesse sentido, destaca-se a importância do fortalecimento e equalização dos princípios e diretrizes da rede de Atenção Básica em municípios fronteiriços, em ambos os lados da fronteira, a fim de garantir o acesso universal e a resolutividade, evitando maiores sequelas para a população.

\section{Contribuições dos autores}

Pereira E participou da concepção e desenho da pesquisa, na coleta, análise e interpretação dos dados, na análise estatística, na redação do manuscrito e na revisão crítica do manuscrito quanto ao conteúdo intelectual importante. Carvalho M participou concepção e desenho da pesquisa, na análise e interpretação dos dados, na análise estatística, na redação do manuscrito e na revisão crítica do manuscrito quanto ao conteúdo intelectual importante.

\section{Conflito de interesses}

Nenhum conflito financeiro, legal ou político envolvendo terceiros (governo, empresas e fundações privadas, etc.) foi declarado para nenhum aspecto do trabalho submetido (incluindo, mas não se limitando a subvenções e financiamentos, participação em conselho consultivo, desenho de estudo, preparação de manuscrito, análise estatística, etc.).

\section{Referências}

1. Pinho NA, Silva GV, Pierin AMG. Prevalência e fatores associados à doença renal crônica em pacientes internados em um hospital universitário na cidade de São Paulo, SP, Brasil. J. Bras. Nefrol. 2015;37(1):91-7. http://dx.doi.org/10.5935/01012800.20150013

2. Draibe AS, organizador. Panorama da Doença Renal Crônica no Brasil e no mundo [Internet]. São Luís: Unasus/UFMA; 2014. Disponível em: https://ares.unasus.gov.br/acervo/handle/ ARES/2028?show=full

3. Souza EBC, Gemelli V. Território, região e fronteira: análise geográfica integrada da fronteira Brasil-Paraguai. Rev. Bras. Estudos Urbanos e Regionais. 2011;13(2):101-16. https://doi. org/10.22296/2317-1529.2011v13n2p101

4. Lei Federal n. 13.445, de 24 de maio de 2017 (Brasil). Lei de Migração. Dispõe sobre os direitos e os deveres do migrante e do visitante, regula a sua entrada e estada no País e estabelece princípios e diretrizes para as políticas públicas para o emigrante [Internet]. Brasília, 24 maio 2017. Disponível em: http://www. planalto.gov.br/ccivil_03/Ato2015-2018/2017/Lei/L13445.htm
5. Zaslavsky R, Goulart BNG. Migração pendular e atenção à saúde na região de fronteira. Ciênc. saúde coletiva. 2017;22(12):3981-6. https://doi.org/10.1590/1413-812320172212.03522016

6. Oliveira MAM, Loio JAM. Migração internacional pendular em fronteira: em busca de qualificações espaciais. Revista Videre. 2019;11(21):54-67. https://doi.org/10.30612/videre.v11i21.9069

7. Decreto $n^{\circ} 7.508$, de 28 de junho de 2011. Regulamenta a Lei $n^{\circ} 8.080$, de 19 de setembro de 1990, para dispor sobre a organização do Sistema Único de Saúde (SUS), o planejamento da saúde, a assistência à saúde e a articulação interfederativa, e dá outras providências. Disponível em: http://www.planalto.gov.br/ ccivil_03/_Ato2011-2014/2011/Decreto/D7508.htm

8. Guerra K, Ventura M. Bioética, imigração e assistência à saúde: tensões e convergências sobre o direito humano à saúde no Brasil na integração regional dos países. Cad. saúde colet. 2017;25(1):123-9. https://doi.org/10.1590/1414$462 \times 201700010185$

9. Aikes S, Rizzotto MLF. Integração regional em cidades gêmeas do Paraná, Brasil, no âmbito da saúde. Cad. Saúde Pública. 2018;34(8):e00182117. https://doi.org/10.1590/0102$\underline{311 \times 00182117}$

10. Silva VR, Ugoski DR, Dravanz GMG. Negação de direitos socioassistenciais para transfronteiriços indocumentados: desafios para as cidades gêmeas. Textos Contextos [Internet]. 2017;16(1):231-43. Disponível em: https://revistaseletronicas. pucrs.br/ojs/index.php/fass/article/view/27121

11. Albuquerque JLC. Limites e paradoxos da cidadania no território fronteiriço: o atendimento dos brasiguaios no sistema público de saúde em Foz do Iguaçu (Brasil). Geopolítica(s). 2012;3(2):185-205. Disponível em: https://revistas.ucm.es/index. php/GEOP/article/viewFile/40040/40396

12. Agencia de Información Paraguaya. Analizarán situación de la nefrología en Paraguay en congreso médico [Internet]. Assunção: Ministerio de Tecnologías de la Información y Comunicación; 2015. Disponível em: http://www.ip.gov.py/ip/?p=52195http:// www.ip.gov.py/ip/?p=52195

13. Campos CEA, Freitas R, Brandão AL, Kastrup E. Cooperação Técnica entre Brasil e Paraguai para a implantação do Programa Saúde da Família no Paraguai [Internet]. Brasília: OPAS; 2013. Disponível em: https://www.paho.org/bra/dmdocuments/ BOASPRATICAS ED6 web.pdf

14. Sesso RC, Lopes AA, Thomé FS, Lugon JR, Martins CT. Inquérito Brasileiro de Diálise Crônica 2016. J. Bras. Nefrol. 2017;39(3):2616. http://dx.doi.org/10.5935/0101-2800.20170049

15. Freitas EB, Bassoli FA, Vanelli CP. Perfil Sociodemográfico de indivíduos portadores de doença renal crônica em tratamento dialítico: estudo descritivo. HU Rev [Internet]. 2014;39(1/2). Disponível em: https://periodicos.ufjf.br/index.php/hurevista/ article/view/2023 
16. Oliveira CS, Silva EC, Ferreira LW, Skalinski LM. Perfil dos pacientes renais crônicos em tratamento hemodialítico. Rev Baiana de Enfermagem [Internet]. 2015;29(1):42-9. Disponível em: https://portalseer.ufba.br/index.php/enfermagem/article/ viewFile/12633/9541

17. Sousa F, Resende V, Silva W, Silva PG, Sousa B, Soares B, et al. Perfil clínico e sociodemográfico de pacientes hemodialisados. Rev. Enferm. Atual In Derme [Internet]. 2020;93(31):e-20039. Disponível em: http://revistaenfermagematual.com.br/index.php/ revista/article/view/643

18. Santos KK, Lucas TC, Glória JCR, Pereira-Júnior AC, Ribeiro GC, Lara MO. Perfil epidemiológico de pacientes renais crônicos em tratamento. Rev de Enfermagem Ufpe on line. 2018;9(2):2293-2300. https://doi.org/10.5205/1981-8963v12i9a234508p2293-2300-2018

19. Ministério da Saúde (Brasil). Diretrizes clínicas para o cuidado ao paciente com doença renal crônica no sistema único de saúde. Brasília: Ministério da Saúde; 2014. Disponível em: https:// bvsms.saude.gov.br/bvs/publicacoes/diretrizes clinicas cuidado paciente_renal.pdf
20. Ministério da Saúde (Brasil). Universidade Federal do Rio Grande do Sul. Protocolos de encaminhamento da atenção básica para a atenção especializada: endocrinologia e nefrologia [Internet]. Brasília: Ministério da Saúde; 2015. Disponível em: http://bvsms.saude.gov.br/bvs/publicacoes/protocolos_atencao basica_atencao_especializada_endocrinologia.pdf

21. Santos JFM, Luz MS, Silva RL, Aguiar V, Moura I, Silva P. Estratégias da atenção básica na doença renal crônica: a importância do diagnóstico precoce. Saúde.com. 2017;13(2):86370. Disponível em: http://periodicos2.uesb.br/index.php/rsc/ article/view/466

22. Abreu LA, Carvalho GAB, Azeredo BA, Mangabeira RR, Souza DC, Guimarães FP, et al. Importância do diagnóstico precoce da Doença Renal Crônica: uma revisão de literatura. Revista Atenas Higeia [Internet]. 2019;1(2):19-23. Disponível em: https://bit. Iy/3mQDTgA 